\title{
Liposomal bupivacaine-a new tool in our armamentarium?
}

\author{
Thomas W. Hamilton' ${ }^{1}$, Hemant G. Pandit ${ }^{1,2,3}$ \\ ${ }^{1}$ Nuffield Department of Orthopaedics, Rheumatology and Musculoskeletal Sciences, University of Oxford, Oxford, UK; ${ }^{2}$ Nuffield Orthopaedic \\ Centre, Oxford University Hospitals NHS Foundation Trust, Oxford, UK; ${ }^{3}$ Leeds Institute of Rheumatic and Musculoskeletal Medicine, University \\ of Leeds, Chapel Allerton Hospital, Leeds, UK \\ Correspondence to: Hemant G. Pandit. Nuffield Department of Orthopaedics, Rheumatology and Musculoskeletal Sciences, University of Oxford, UK; \\ Nuffield Orthopaedic Centre, Oxford University Hospitals NHS Foundation Trust, Oxford, UK. Email: hemant.pandit@ndorms.ox.ac.uk.
}

Received: 10 September 2016; Accepted: 23 September 2016; Published: 04 November 2016.

doi: 10.21037/aoj.2016.10.01

View this article at: http://dx.doi.org/10.21037/aoj.2016.10.01

Almost two decades on from when Enhanced Recovery Programs (ERPs), consisting of multi-modal therapies to reduce surgical stress, were first proposed by Kehlet et al. the complexity of ERPs has increased, with interventions now utilized pre-, intra- and post-operatively (1). Whilst the use of ERPs has been demonstrated to significantly reduce patient morbidity and mortality, and reduce costs to the healthcare system, what constitutes the gold standard ERP has yet to be defined and significant variation in practice exists (2). Despite improvements in ERPs pain remains the primary cause of delayed discharge, as well as a common cause of re-admission, following orthopaedic surgery (3).

The ideal analgesia is one that is simple to administer, gives a localized pure sensory block, has a low risk of adverse events and has a duration of action that exceeds the duration of operative pain. Local anesthetic infiltration fulfils many of these criteria, and following lower limb arthroplasty its use has been associated with a reduced analgesia requirement, earlier mobilization and shorter hospital inpatient stays (4). A limitation of local anesthetics however is their duration of action with patients often reporting rebound pain within 24 hours of administration (5). To address this issue, continuous local anesthetic infiltration has been used, however concerns about the risk of infection, as well as the cost of infusion pumps has limited the uptake of this technique.

Liposomal bupivacaine (Exparel, Pacira Pharmaceuticals, USA) was approved by the Food and Drug Administration (FDA) in the United States in October 2011 and has been used in more than 2 million patients for post-operative pain management across a range of surgical specialties (6). It seeks to improve the duration of action of analgesia by utilizing multi-vesicular liposomes to delay the release of local anesthetic (7). Administered as a single intra-operative dose it has the potential to offer a long acting sensory block and as such revolutionize post-operative analgesia.

Liposomal bupivacaine infiltration at the surgical site has been assessed in 13 randomized controlled trials across six surgical sites, including five studies evaluating its use for pain control following total knee replacement (TKR) and is currently the subject of a Cochrane review (8-13). The use of liposomal bupivacaine for pain control following anterior cruciate ligament (ACL) reconstruction has not previously been reported.

Premkumar and colleagues investigated, in patients undergoing quadriceps tendon autograft ACL reconstruction, whether liposomal bupivacaine, $266 \mathrm{mg}$, offered superior analgesia to bupivacaine hydrochloride, $100 \mathrm{mg}$, when administered intra-operatively in combination with a with a femoral nerve block ( 1 to $1.5 \mathrm{mg} / \mathrm{kg}$ of $0.5 \%$ ropivacaine hydrochloride) and multi-modal ERP (14). Utilizing a doubleblind randomized study design, and assessing 29 patients, the authors found no difference in any outcome measures: including post-operative pain at any time point through day 5 , time to first opioid, total opioid use per day through day 5 , cumulative opioid use 0 to 72 hours or time to straight leg raise. In conclusion, given the comparable outcomes and significantly lower cost of bupivacaine hydrochloride, the authors stated there was no evidence to support the use of liposomal bupivacaine for pain control after ACL reconstruction performed under femoral nerve block.

Putting the results of this trial into the context of the current literature it must be noted that, none of the five, active 
comparator, randomized controlled trials (679 participants) that have assessed the role of liposomal bupivacaine following TKR, have demonstrated reduced pain scores, or analgesia consumption, at the licensed dose of $266 \mathrm{mg}$ $(8-11,13)$. Should we therefore conclude that liposomal bupivacaine has no role in ERPs for knee surgery?

Whilst at present we can say that the limited evidence does not demonstrate superiority of liposomal bupivacaine to bupivacaine hydrochloride, we must acknowledge that the majority of trials that have been conducted, including the one by Premkumar et al., are small meaning that further large, well conducted, multi-centre prospective randomized controlled trials have a significant chance of changing our estimate of effect. As our experience with liposomal bupivacaine increases so will our understanding of its role, if any, within orthopaedic surgery. As with all new interventions there is a learning curve and with liposomal bupivacaine recent research has highlighted the importance of injection technique as well questioned whether there is a need to admix liposomal bupivacaine with bupivacaine hydrochloride to achieve rapid onset analgesia $(15,16)$. To what extent these factors influence the efficacy of liposomal bupivacaine remains to be seen, but it is important that these assumptions are tested before firm conclusions are drawn.

Another question that must be answered is whether liposomal bupivacaine is an adjunct or replacement to traditional techniques? In the trial by Premkumar et al. liposomal bupivacaine is used as an adjunct to femoral nerve block and as such we must ask ourselves whether a different result would be seen if a femoral nerve block was not used? In the study by Premkumar et al. it is likely that the femoral nerve block provided analgesia for the first 40 hours, as assessed by inability to straight leg raise, and as such any difference in efficacy of liposomal bupivacaine compared with bupivacaine hydrochloride would only be detectable after this time point. If differences in efficacy between the two drugs does exist before 40 hours then, depending on the magnitude and duration of this difference, different conclusions may be drawn, particularly as the results demonstrate a reduction in opioid requirement from day three onwards.

Finally, we must acknowledge that the current research is focused on pain scores and analgesic consumption, and whilst these factors are important, they do no full encompass factors that patients report as important for their recovery (17). Whilst at the present time, there are no fully validated patient reported tools to assess early postoperative recovery it is known that the absence of nausea, ability to ambulate as well as to perform self-care are important to patients and further work is required to identify what constitutes the optimum recovery from the patients perspective.

Whilst there is currently uncertainty around what constitutes the optimum treatment and also what outcome measures we should be assessing what is certain is that improvements in post-operative pain management will deliver significant benefits to patients, healthcare professionals and healthcare payers. As such innovations such as liposomal bupivacaine, which have the potential to revolutionize care, will continue to emerge and it is important that these are rigorously assessed as to their clinical and cost effectiveness prior to their widespread use.

\section{Acknowledgements}

Thomas W. Hamilton has been supported by the NIHR Biomedical Research Centre, based at Oxford University Hospitals Trust, Oxford.

\section{Footnote}

Conflicts of Interest: The authors have no conflicts of interest to declare.

Disclaimer: The views expressed are those of the author(s) and not necessarily those of the NHS, the NIHR or the Department of Health.

\section{References}

1. Kehlet H. Multimodal approach to control postoperative pathophysiology and rehabilitation. Br J Anaesth 1997;78:606-17.

2. Nagra NS, Hamilton TW, BONE Collaboration, et al. Inconsistencies in the Enhanced Recovery Programmes across the NHS. Telford, UK: British Association for Surgery of the Knee, 2015 Annual Spring Meeting, 1011th March, 2015.

3. Husted H, Lunn TH, Troelsen A, et al. Why still in hospital after fast-track hip and knee arthroplasty? Acta Orthop 2011;82:679-84.

4. Marques EM, Jones HE, Elvers KT, et al. Local anaesthetic infiltration for peri-operative pain control in total hip and knee replacement: systematic review and meta-analyses of short- and long-term effectiveness. BMC Musculoskelet Disord 2014;15:220. 
5. Wei J, Lei GH, Gao SG, et al. Single-dose intra-articular bupivacaine versus morphine after arthroscopic knee surgery: a meta-analysis of randomized-controlled studies. Clin J Pain 2014;30:630-8.

6. Home page of Pacira Pharmaceuticals. Available online: http://www.exparel.com

7. Mantripragada S. A lipid based depot (DepoFoam technology) for sustained release drug delivery. Prog Lipid Res 2002;41:392-406.

8. Bramlett K, Onel E, Viscusi ER, et al. A randomized, double-blind, dose-ranging study comparing wound infiltration of DepoFoam bupivacaine, an extended-release liposomal bupivacaine, to bupivacaine $\mathrm{HCl}$ for postsurgical analgesia in total knee arthroplasty. Knee 2012;19:530-6.

9. Collis PN, Hunter AM, Vaughn MD, et al. Periarticular Injection After Total Knee Arthroplasty Using Liposomal Bupivacaine vs a Modified Ranawat Suspension: A Prospective, Randomized Study. J Arthroplasty 2016;31:633-6.

10. Surdam JW, Licini DJ, Baynes NT, et al. The use of exparel (liposomal bupivacaine) to manage postoperative pain in unilateral total knee arthroplasty patients. J Arthroplasty 2015;30:325-9.

11. Schroer WC, Diesfeld PG, LeMarr AR, et al. Does Extended-Release Liposomal Bupivacaine Better Control Pain Than Bupivacaine After Total Knee Arthroplasty (TKA)? A Prospective, Randomized Clinical Trial. J Arthroplasty 2015;30:64-7.

12. Hamilton TW, Athanassoglou V, Mellon S, et al.

doi: 10.21037/aoj.2016.10.01

Cite this article as: Hamilton TW, Pandit HG. Liposomal bupivacaine-a new tool in our armamentarium? Ann Joint 2016;1:22.
Liposomal bupivacaine infiltration at the surgical site for the management of postoperative pain. Available online: Liposomal bupivacaine infiltration at the surgical site for the management of postoperative pain

13. A Phase 3 Study to Evaluate the Safety and Efficacy of SKY0402 in Subjects Undergoing Total Knee Arthroplasty (TKA). Available online: https://clinicaltrials.gov/ct2/ show/NCT00745290

14. Premkumar A, Samady H, Slone H, et al. Liposomal Bupivacaine for Pain Control After Anterior Cruciate Ligament Reconstruction: A Prospective, Double-Blinded, Randomized, Positive-Controlled Trial. Am J Sports Med 2016;44:1680-6.

15. Joshi GP, Cushner FD, Barrington JW, et al. Techniques for periarticular infiltration with liposomal bupivacaine for the management of pain after hip and knee arthroplasty: a consensus recommendation. J Surg Orthop Adv 2015;24:27-35.

16. Gadsden J, Long WJ. Time to Analgesia Onset and Pharmacokinetics After Separate and Combined Administration of Liposome Bupivacaine and Bupivacaine $\mathrm{HCl}$ : Considerations for Clinicians. Open Orthop J 2016;10:94-104.

17. Strickland LH, Hamilton TW, Jenkinson CC, et al. Patient-Reported Outcome Measure for Early Postoperative Recovery Following Lower Limb Arthroplasty: A Systematic Review. J Arthroplasty 2016. [Epub ahead of print]. 